\title{
A prospective study of dual-energy computed tomography for differentiating metastatic and non-metastatic lymph nodes of colorectal cancer
}

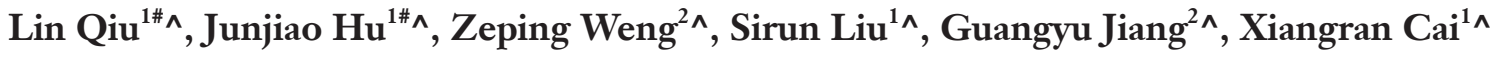 \\ ${ }^{1}$ Medical Imaging Center, The First Affiliated Hospital of Jinan University, Guangzhou, China; ${ }^{2}$ Pathology Department, The First Affiliated Hospital \\ of Jinan University, Guangzhou, China
}

Contributions: (I) Conception and design: X Cai; (II) Administrative support: X Cai; (III) Provision of study materials or patients: L Qiu, J Hu, Z Weng; (IV) Collection and assembly of data: L Qiu, J Hu; (V) Data analysis and interpretation: L Qiu, J Hu, G Jiang, S Liu; (VI) Manuscript writing: All authors; (VII) Final approval of manuscript: All authors.

\#These authors contributed equally to this work.

Correspondence to: Xiangran Cai. Medical Imaging Center, The First Affiliated Hospital of Jinan University, No. 613 West Huangpu Avenue, Tianhe District, Guangzhou 510630, China. Email: caixran@jnu.edu.cn.

\begin{abstract}
Background: Colorectal cancer (CRC) is the third most common malignancy worldwide, and lymph node metastasis is considered to be a risk factor for local recurrence and a poor prognosis in colorectal cancer. However, there remains a lack of reliable and non-invasive biomarkers to identify the lymph node status of CRC patients preoperatively. The purpose of this study was to explore the ability of dual-energy computed tomography (DECT) to differentiate metastatic from non-metastatic lymph nodes in colorectal cancer.

Methods: Seventy-one patients with primary colorectal cancer underwent contrast-enhanced dual-energy computed tomography imaging preoperatively. The colorectal specimen was scanned postoperatively, and lymph nodes were matched to the pathology report. The following dual-energy computed tomography quantitative parameters were analyzed: dual-energy curve slope value $\left(\lambda_{\mathrm{HU}}\right)$, standardized iodine concentration ( $\triangle H U$ ), iodine water ratio (nIWR), electron density value (n $\rho_{\text {eff }}$ ), and effective atom-number $(\mathrm{nZ})$, based on metastatic and non-metastatic lymph node differentiation. Also, sensitivity and specificity analyses were performed using receiver operating characteristic curves.

Results: In all patients, one hundred and fifty lymph nodes, including 66 non-metastatic and 84 metastatic lymph nodes, were matched using the radiological-pathological correlation. Metastatic nodes had significantly greater $\lambda_{\mathrm{HU}}, \mathrm{n} \triangle \mathrm{HU}$, and nIWR values than non-metastatic nodes in both the arterial and venous phases $(\mathrm{P}<0.01)$. The area under curve (AUC), sensitivity, and specificity were $0.80,80 \%$, and $66 \%$ for $\lambda_{\mathrm{HU}} ; 0.86,70 \%$, and $95 \%$ for $\mathrm{n} \triangle \mathrm{HU}$; and $0.88,71 \%$, and $95 \%$ for nIWR in the arterial phase. There was no significant difference in electron density and effective $Z$ values between metastatic and non-metastatic lymph nodes.
\end{abstract}

Conclusions: DECT quantitative parameters may help differentiate between metastatic and normal lymph nodes in patients with CRC.

Keywords: Computed tomography; dual-energy CT (DECT); colorectal cancer (CRC); lymph node; staging

\footnotetext{
^ ORCID: Xiangran Cai, 0000-0003-4010-7577; Lin Qiu, 0000-0001-8423-1751; Junjiao Hu, 0000-0002-9005-3696; Zeping Weng, 00000002-4435-4316; Sirun Liu, 0000-0002-6567-524X; Guangyu Jiang, 0000-0002-7350-8732.
} 
Submitted Jan 01, 2020. Accepted for publication Apr 28, 2021.

doi: $10.21037 /$ qims-20-3

View this article at: http://dx.doi.org/10.21037/qims-20-3

\section{Introduction}

Colorectal cancer (CRC) is the third most common malignancy and the fourth leading cause of cancer-related death in the Western world (1). Over the past 10 years, 1.2 million new CRC cases were recorded $(2,3)$. Although its incidence in China is lower than that in Western countries, the prevalence of CRC has increased due to lifestyle changes in recent years and has become a substantial cancer burden in China, particularly in urban areas (4). Lymph node metastasis is considered to be a risk factor for local recurrence and a poor prognosis in CRC (5). Numerous studies have revealed that the 5 -year overall survival rate was $80-90 \%$ for CRC patients without lymph node metastasis, compared to $60-68 \%$ for those with nodal metastasis (6-8). A higher local recurrent rate is found in CRC patients with nodal metastasis than in patients with negative nodes (9). Preoperative neoadjuvant therapy has been recommended by the National Comprehensive Cancer Network (NCCN) for patients with positive lymph nodes, significantly reducing the risk of local recurrence and improving the survival rate (10). Therefore, accurate preoperative detection of lymphatic metastasis is essential for selecting further treatment for these patients.

High-resolution magnetic resonance (MR) imaging has been employed in an attempt to detect evidence of lymph node metastasis in CRC (11). Also, functional MR techniques have been used to evaluate the lymph node status in CRC, such as diffusion-weighted imaging (DWI). Qiu et al. applied intravoxel incoherent motion imaging (IVIM) for differentiating metastatic from non-metastatic nodes in patients with rectal carcinoma (12) and found IVIM parameters may be helpful for differentiation. However, due to the limited spatial resolution of IVIM images and the discrepant b-value numbers distribution in different studies (13), this method cannot be directly used to predict nodal involvement in clinical practice.

Dual-energy CT (DECT) is an advanced CT technique that evaluates tissues at different $\mathrm{X}$-ray energies, enabling spectral evaluation and material tissue characterization (14). It can reconstruct material decomposition images and a set of monochromatic images with photon energy ranging from 40 to $140 \mathrm{keV}$ to generate spectral Hounsfield (HU) curves and obtain multiple quantitative parameters (15).
We hypothesize that these parameters could preoperatively distinguish malignant from benign lymph nodes in CRC. Indeed, various studies have increasingly demonstrated the potential advantages of DECT for the evaluation of lung adenocarcinoma (16), lymph node metastasis in gastric cancer (17), liver lesions (18-20), pancreatic tumor (21), and the prediction of metastatic lymph nodes in head and neck cancer (22). Nevertheless, the application of DECT in the diagnosis of lymphatic metastases in CRC has rarely been reported. The present study was designed to investigate whether the parameters of biphasic contrast-enhanced DECT are beneficial in the differentiation of metastatic from non-metastatic lymph nodes in CRC.

\section{Methods}

\section{Patients}

This study was conducted following the Declaration of Helsinki (as revised in 2013) and was approved by the Medical Ethics Committee of the First Affiliated Hospital of Jinan University. All patients signed an informed consent after having the nature of the study fully explained to them. Ninety consecutive patients with primary CRC were enrolled from May 2015 to March 2019. The inclusion criteria were as follows: (I) patients confirmed as CRC by biopsy; (II) patients who did not receive radiotherapy or chemotherapy before surgery; and (III) patients with no allergic iodine history or symptoms of hyperthyroidism. Of the 90 patients initially enrolled, 19 cases were excluded due to the following reasons: (I) poor preparation of bowel $(\mathrm{n}=7)$; (II) poor intestinal filling $(\mathrm{n}=5)$; (III) metal artifacts caused by pelvic or lumbar surgery ( $n=3$ ); and (IV) presence of gas artifacts affecting observation $(n=4)$. Ultimately, 71 cases were included in this study, including 41 males and 30 females, aged 31-91 years, with a mean age of $(59.3 \pm 14.1)$ years. Patients underwent dual-phasic contrastenhanced DECT 1-3 days before surgery.

\section{DECT scan}

The day before the examination, patients were given a light diet that does not easily produce gas and dregs. On the morning of the DECT examination, patients received 
a cleansing enema in the ward until a watery stool was discharged. 400-1,000 $\mathrm{mL}$ warm water (according to patients' tolerance) was injected into the rectum before scanning.

All examinations were performed using a single-source CT system (Aquilion ONE; Canon Medical Systems, Otawara, Tochigi, Japan), equipped with 320-detector raw and covered $160 \mathrm{~mm}$ in the z-direction. This system can rapidly switch tube voltage from 80 to $135 \mathrm{kV}$ within 50 milliseconds and automatically adapt the corresponding tube current. All patients were scanned in the supine position and underwent a routine non-contrast CT scanning with the following scanning parameters: tube voltage, $140 \mathrm{kV}$; tube current, $112-187 \mathrm{~mA}$; matrix, $512 \times 512$; field of view (FOV), $240 \mathrm{~mm}$; rotation speed, $0.35 \mathrm{~s} /$ rot; slice thickness, $0.5 \mathrm{~mm}$; slice spacing, $0.5 \mathrm{~mm}$; and scanning scope ranging from the diaphragmatic dome to the pubic symphysis. Dual-phasic contrast-enhanced scans were then performed using the dual-energy mode. The scan parameters were as follows: matrix, $512 \times 512$; FOV, $240 \mathrm{~mm}$; rotation speed, $0.35 \mathrm{~s} / \mathrm{rot}$; and slice thickness, $0.5 \mathrm{~mm}$; and volume scan mode. $55-86 \mathrm{~mL}(1.0 \mathrm{~mL} / \mathrm{kg})$ of a non-ionic iodinated contrast agent (Ultravist300; Schering, Berlin, Germany) was administered via the antecubital vein at a flow rate of $3 \mathrm{~mL} / \mathrm{s}$ by G22 needle (BD Inrima$\mathrm{II}^{\mathrm{TM}}$; Becton Dickinson and Company, Suzhou Industrial Park, Jiangsu, China) through an automatic injector. Dualphasic scans were obtained at $40 \mathrm{~s}$ (arterial phase) and $70 \mathrm{~s}$ (venous phase) centering the tumor after the start of the contrast injection, respectively. The third generation AIDR 3D iterative algorithm developed by Canon was used, and the effective dose was $5.39 \pm 0.48 \mathrm{mSv}$.

\section{Histological examination}

After surgical resection of the colorectal tumor, each specimen was taken back to the Department of Radiology and imaged again using the same conventional non-contrast sequence in the coronal and transverse planes before the specimen was fixed with formalin. After scanning, the specimens were immediately fixed with $4 \%$ neutral formaldehyde and delivered for pathological examination.

The image slices of the preoperative CT scan and the postoperative specimens were compared to locate and mark all correct topographical matching lymph nodes with a diameter greater than $4 \mathrm{~mm}$ in a blinded fashion by two radiologists (LQ and XRC, with 9 and 20 years of abdominal imaging experience, respectively) in consensus.
The morphological features of each lymph node were recorded according to the CT characteristics, i.e., shortaxis diameter, shape, border, and density. Subsequently, the specimens were delivered to the Department of Pathology in order to improve the matching rate of lymph nodes between the DECT images and pathological specimens, where every marked lymph node was harvested by one radiologist (LQ) and one pathologist (ZPW, with 10 years of experience in pathological diagnosis), who were blinded to the clinical and biochemical data of all patients. Any mismatch between specimen CT images and the histological findings were recorded and excluded from the study. Finally, all good-matched lymph nodes were stained with hematoxylin \& eosin and placed under a microscope (Olympus BX43; Tokyo, Japan) to observe histological features by two pathologists (ZPW and GYJ, with 20 years of experience in pathological diagnosis) to determine the presence of tumor deposit.

\section{Data analysis}

The dual-phasic image volumes were loaded into the DECT postprocessing software in the display console (Canon Aquilion ONE, Canon Medical System). The DECT software automatically generated 35-135 Kev CT values to form a single-energy CT curve, dual-energy iodine, base material, electron density, and atom number images. A region of interest (ROI) of $12-78 \mathrm{~mm}^{2}$ was drawn using a circular tool on each marked lymph node on the reconstructed $69-\mathrm{keV}$ monochromatic images (Figure 1). The ROI was chosen to cover the nodal parenchyma as much as possible. The obvious suspicious necrotic region of fatty hilum was avoided from the ROI to minimize the influence of potential errors. Each lymph node was measured three times, and a mean value was calculated. Another ROI was placed on the right iliac artery at the corresponding layer as a reference. All of the ROIs were automatically copied onto all dual-energy iodine, base material, electron density, and atom number images to calculate iodine concentration value $(\triangle \mathrm{HU})$, iodine water ratio value (IWR), electron density value $\left(\rho_{\text {eff }}\right)$, and an atom number value $(Z)$. Measurements were performed in a blinded fashion by another two independent radiologists.

\section{Statistical analysis}

The sample size was calculated using PASS software version 15.0 (NCSS, Kaysville, Utah, USA). Under the 

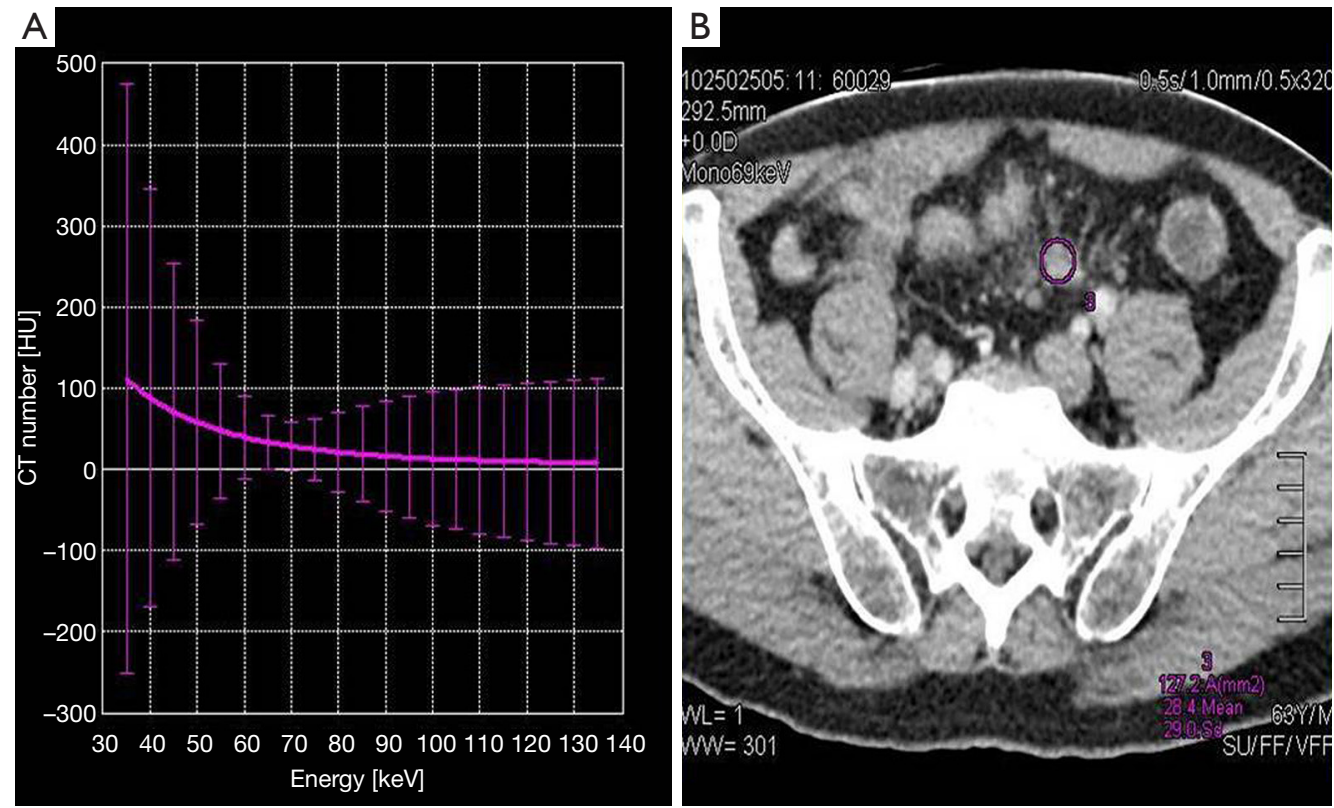

Figure 1 Measurement of lymph nodes; (A) dual-energy CT curve; (B) 69-KeV monochromatic image. Measurement using the tools contained in the system. ROI was selected on the lymph node and included the entire lymph node as much as possible. ROIs, regions of interest.

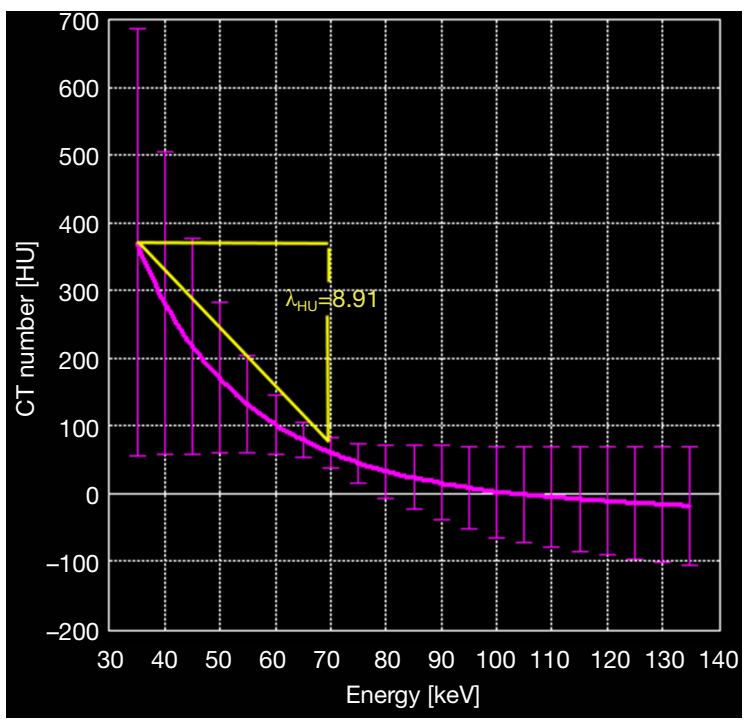

Figure 2 The method of $\lambda_{\mathrm{Hu}}$ calculation. The calculation method for the slope of the ROI curve is shown. The pink line is a dualenergy CT curve; the slope of the curve in the ROI is 8.91. $\lambda_{\mathrm{Hu}}$, dual-energy curve slope value. receiver operator characteristic (ROC) curve (AUC), the hypothesized area was 0.7 with 0.5 for null hypothesis value. The rate of block failure was estimated to be $30 \%$, according to our previous pilot study. Setting the $\alpha$ level as 0.05 and the power as $90 \%$, the minimum required sample size was 82 , with 41 malignant and 41 normal lymph nodes. We decided to include more than 107 lymph nodes to account for dropout.

The slope of the dual-energy curve was expressed as $\lambda_{\mathrm{HU}}(23)$, which was calculated by $\lambda_{\mathrm{HU}}=\left(35 \mathrm{KeV}_{\mathrm{HU}}-\right.$ $\left.69 \mathrm{KeV}_{\mathrm{HU}}\right) / 34 \mathrm{KeV}$, where $35 \mathrm{KeV}_{\mathrm{HU}}$ and $69 \mathrm{KeV}_{\mathrm{HU}}$ were the $\mathrm{CT}$ values at $35 \mathrm{KeV}$ and $69 \mathrm{KeV}$, respectively (Figure 2). The $\triangle H U, I W R, \rho_{\text {eff, }}$ and $Z$ values of the lymph nodes were normalized to values in the iliac artery to drive the normalized values: $\mathrm{n} \Delta \mathrm{HU}=\Delta \mathrm{HU}_{\text {tissue }} / \triangle H \mathrm{U}_{\text {artery }}$, $\mathrm{nIWR}=\mathrm{IWR}_{\text {tissue }} / \mathrm{IWR}_{\text {artery }}, \mathrm{n} \rho_{\text {eff }}=\rho_{\text {efftissue }} / \rho_{\text {effartery }}$ and $\mathrm{nZ}=Z_{\text {tissue }} /$ $Z_{\text {artery. }}$ Measurements were carried out separately by two observers, and the average values were obtained for statistical analysis.

Statistical analyses were performed using SPSS20.0 (IBM, 

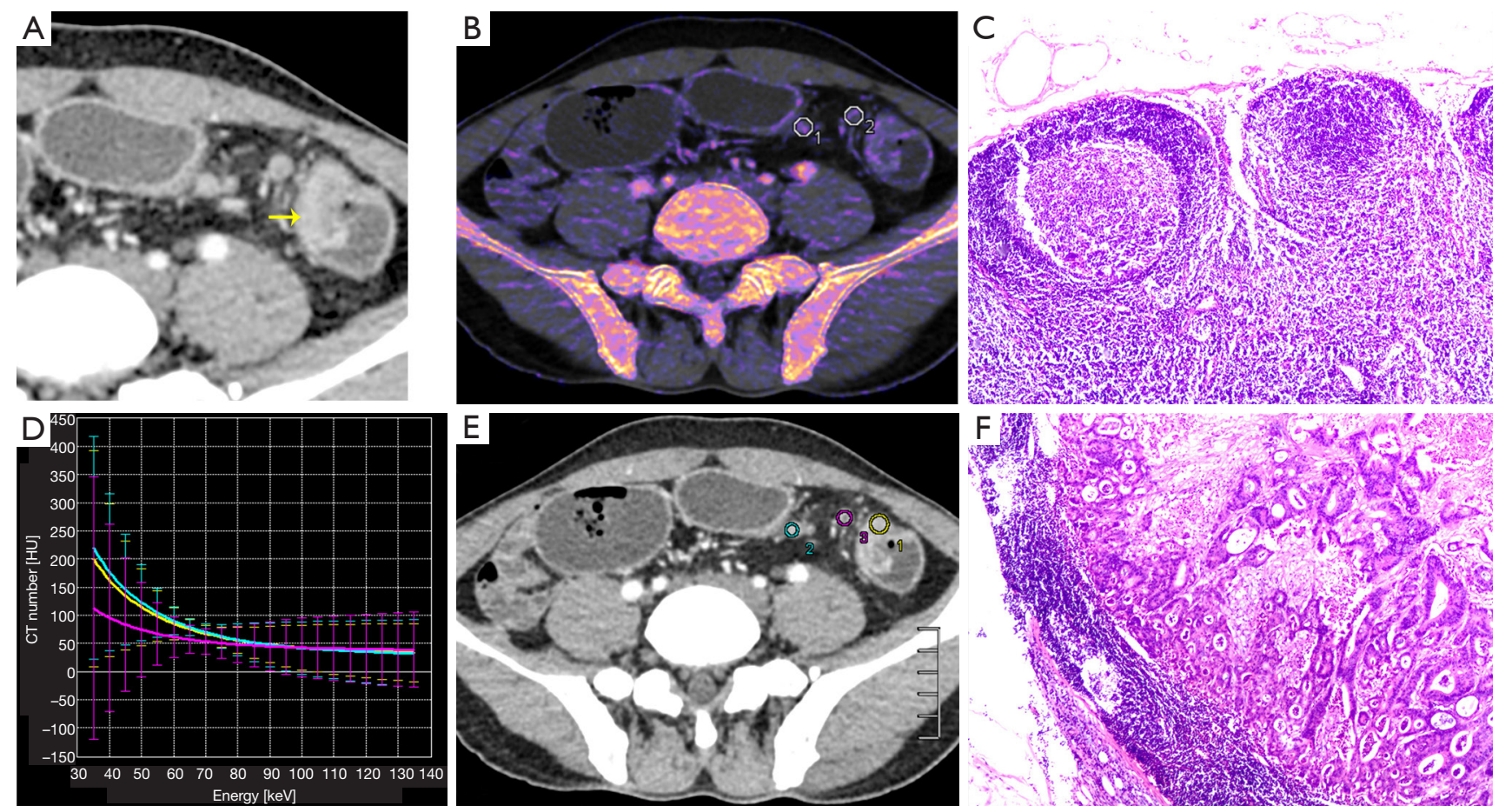

Figure 3 A 41-year-old man with colon cancer; (A) monochromatic obtained at $69 \mathrm{Kev}$ energy level with the best signal-to-noise ratio: a mass shallow was visible in the descending colon (yellow arrow), with multiple lymph nodes of different sizes; (B) dual-energy iodine image; (C) representative images of hematoxylin and eosin $(\mathrm{H} \& \mathrm{E})$ stains of normal lymph nodes $(\times 40)$; $(\mathrm{D}, \mathrm{E})$ dual-energy curve: the dual energy curve of the tumor (yellow line) almost overlaps that of metastatic lymph node (cyan line), with the same slope. The slope of non-metastatic lymph nodes (pink line) was smaller than that of the metastatic lymph node; (F) representative images of the hematoxylin and eosin (H\&E) stains of metastatic lymph nodes of adenocarcinoma, demonstrating gland formation by malignant cells $(\times 40)$. DECT parameters were used to differentiate normal and metastatic lymph nodes.

Armonk) and Medcalc (MedCalc Software, Acacialaan 22, B-8400) software. A difference with $\mathrm{P}<0.05$ was considered statistically significant. Measurement data following normal distribution were expressed by $\bar{x} \pm \mathrm{s}$. Bland-Altman plots and the intraclass correlation coefficient (ICC) were used to analyze the interobserver variability of $\lambda_{\mathrm{HU}}, \mathrm{n} \triangle \mathrm{HU}, \mathrm{nIWR}$, $\mathrm{n} \rho_{\text {eff }}$, and $\mathrm{nZ}$ values: poor (less than 0.40 ), fair (0.40-0.59), good (0.60-0.74), and excellent (0.75-1.00). The DECT parameters for the non-metastatic and metastatic lymph nodes were analyzed separately using the two-sample $t$-test. The short-axis diameter and the DECT parameters with statistical significance were used to plot receiver operating characteristic (ROC) curves, in which the optimal cut-off values were obtained using the Maximum Youden's index method.

\section{Results}

\section{Histopathological results and morphological features}

All patients received laparoscopic surgical excision. There were 43 patients with moderately differentiated adenocarcinoma and 28 patients with poorly differentiated adenocarcinoma in terms of the histological results. A total of 399 peritumoral lymph nodes were harvested via histopathology. Of these, 249 normal nodes (less than $4 \mathrm{~mm}$ in diameter) were excluded due to mismatch. Therefore, the remaining 150 lymph nodes around the tumor were available for analysis, of which 84 lymph nodes were malignant, and 66 were normal. Figure 3 shows representative images of the normal and metastatic nodes.

There were more metastatic lymph nodes in those with 
Table 1 CT Features in 150 lymph nodes

\begin{tabular}{|c|c|c|c|c|c|c|c|}
\hline CT imaging features & $\begin{array}{l}\text { Lymph nodes } \\
\text { (n) }\end{array}$ & $\begin{array}{c}\text { Metastatic } \\
\text { nodes }(n)\end{array}$ & $\begin{array}{c}\text { Prevalence } \\
(\%)\end{array}$ & $\begin{array}{c}\text { Non-metastatic } \\
\text { nodes }(n)\end{array}$ & $\begin{array}{c}\text { Prevalence } \\
(\%)\end{array}$ & $\chi^{2}$ & $P$ value \\
\hline \multicolumn{8}{|l|}{ Size } \\
\hline $4 \mathrm{~mm} \geq \mathrm{D} \leq 5 \mathrm{~mm}$ & 17 & 7 & 41.18 & 10 & 58.82 & & \\
\hline $5 \mathrm{~mm}<\mathrm{D} \leq 10 \mathrm{~mm}$ & 108 & 56 & 51.85 & 52 & 48.15 & 11.53 & $<0.01$ \\
\hline \multicolumn{8}{|l|}{ Shape } \\
\hline Round & 97 & 48 & 49.48 & 49 & 50.52 & 4.73 & 0.03 \\
\hline Elliptical & 53 & 36 & 67.92 & 17 & 32.08 & & \\
\hline \multicolumn{8}{|l|}{ Border } \\
\hline \multicolumn{8}{|l|}{ Density $^{\dagger}$} \\
\hline Homogeneous & 110 & 54 & 49.09 & 56 & 50.91 & 7.99 & 0.01 \\
\hline Heterogeneous & 40 & 30 & 75.00 & 10 & 25.00 & & \\
\hline \multicolumn{8}{|l|}{ T staging } \\
\hline $\mathrm{T}_{3}$ staging & 117 & 58 & 49.57 & 59 & 50.43 & 8.92 & $<0.01$ \\
\hline $\mathrm{T}_{4}$ staging & 33 & 26 & 78.79 & 7 & 21.21 & & \\
\hline \multicolumn{8}{|l|}{ Differentiation } \\
\hline Poorly & 63 & 46 & 73.02 & 17 & 26.98 & 12.76 & $<0.01$ \\
\hline
\end{tabular}

${ }^{\dagger}$ Density on the reconstructed $69-\mathrm{keV}$ monochromatic images of the arterial phase. $\mathrm{D}$, the maximal short-axis diameter.

greater than $10 \mathrm{~mm}$ in short-axis diameter. Also, more metastatic nodes were heterogeneous. Also, more metastatic nodes were found in patients with T4 staging and poorly differentiated CRC. Table 1 shows the distribution of all normal and metastatic lymph nodes regarding the shortaxis, morphology, and pathology.

\section{Interobserver agreement}

Measurements by observers 1 and 2 for normal and metastatic lymph nodes are summarized in Table 1. Excellent interobserver agreement was seen between the two observers in measuring all DECT parameters (Table 2). The Bland-Altman plots for nodal measurements are displayed in Figure 4. In the Bland-Altman plots, the points tended to be distributed around the mean difference line, and most of the values were included within the range of 1.96 SD (Standard Deviation) of the difference.

\section{Dual-energy characteristics of metastatic and non- metastatic lymph nodes}

The $\lambda_{\mathrm{HU}}, \mathrm{n} \triangle \mathrm{HU}$, and $\mathrm{nIWR}$ values of metastatic nodes in the arterial and venous phases were significantly greater than those of non-metastatic nodes $(\mathrm{P}<0.01)$. However, $n \rho_{\text {eff }}$ and $\mathrm{nZ}$ did not show significant differences between the metastatic and non-metastatic lymph nodes (Table 3).

The ROC curves of the DECT parametric values $\left(\lambda_{\mathrm{HU}}, \mathrm{n} \triangle \mathrm{HU}\right.$, and $\left.\mathrm{nIWR}\right)$ and short-axis diameter in differentiating normal from metastatic lymph nodes are shown in Figure 5 and Table 4. The ROC curves demonstrated that the AUC values of all DECT parameters in the arterial phase were greater than those in the venous phase. Also, the AUC of the nIWR, $\mathrm{n} \triangle \mathrm{HU}$, and $\lambda_{\mathrm{HU}}$ statistically decreased. The nIWR had the highest AUC, while $\lambda_{\mathrm{HU}}$ and short-axis diameter had the lowest. Compared with the DECT parameters, the AUC value of the short 
Table 2 Consistency check results of two observers on each observation in the arterial and venous phases

\begin{tabular}{|c|c|c|c|c|c|}
\hline & $\lambda_{\text {HU }}$ & $\mathrm{n} \triangle \mathrm{Hu}$ & nIWR & $\mathrm{n} \rho_{\text {eff }}$ & $\mathrm{nZ}$ \\
\hline \multicolumn{6}{|l|}{ Arterial phase } \\
\hline ICC & 0.99 & 0.99 & 0.94 & 0.98 & 0.99 \\
\hline $95 \%$ confidence interval & $0.99-0.99$ & $0.99-0.99$ & $0.91-0.95$ & $0.98-0.99$ & $0.99-0.99$ \\
\hline \multicolumn{6}{|l|}{ Venous phase } \\
\hline $95 \%$ confidence interval & $0.99-0.99$ & $0.98-0.99$ & $0.89-0.94$ & $0.93-0.96$ & $0.99-0.99$ \\
\hline
\end{tabular}

$\lambda_{\text {HU }}$, dual-energy curve slope value; $\mathrm{n} \triangle \mathrm{HU}$, standardized iodine concentration; nIWR, iodine water ratio; $n \rho_{\text {eff, }}$ electron density value; $\mathrm{nZ}$, effective atom-number; ICC, intraclass correlation coefficient.
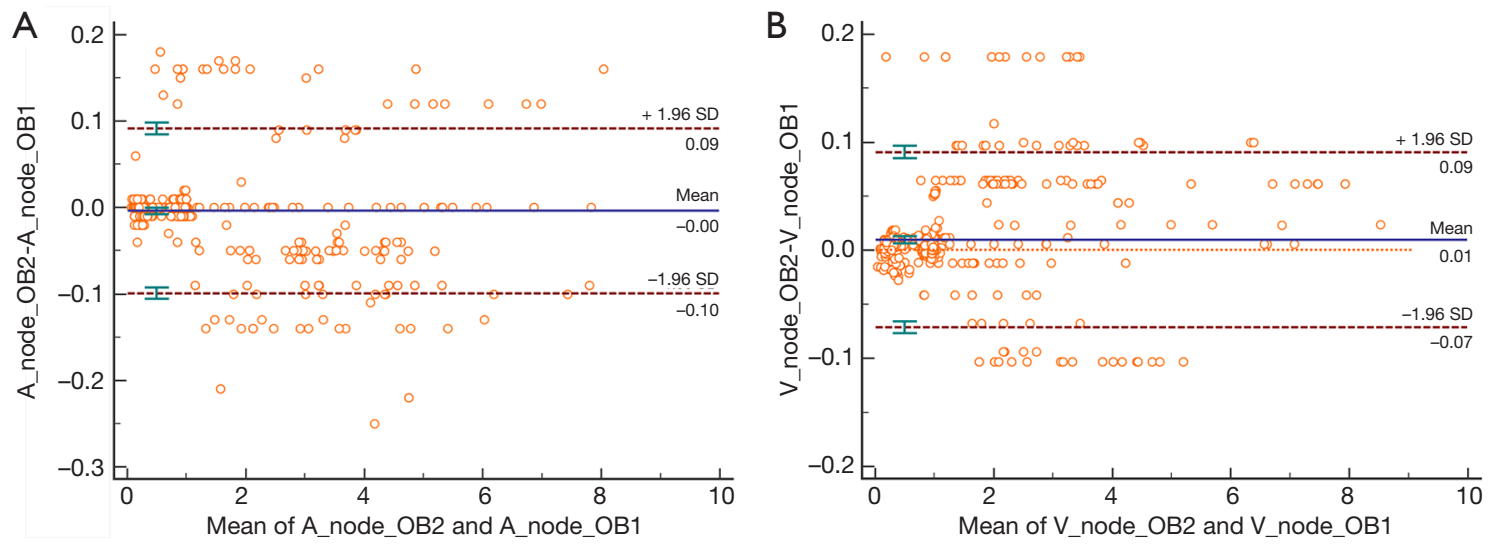

Figure 4 Bland-Altman plots for interobserver agreements of DECT parameters $\left(\lambda_{\mathrm{HU}}, \mathrm{n} \triangle \mathrm{HU}, \mathrm{nIWR}, \mathrm{n} \rho_{\mathrm{eff}}\right.$, and $\left.\mathrm{nZ}\right)$ measurements in the arterial phase (A) and venous phase (B). The X-axis is the average of the DECT parameters values of the two readers and the Y-axis is the percentage difference in the DECT parameters values between the two readers. The solid line shows the mean difference, while the dashed lines show the $95 \%$ limits of agreement. A, arterial phase; V, venous phase; OB, observer. The plot is useful in revealing the relationship between the differences of the two interobservers.

Table 3 Dual-energy parameters of metastatic and non-metastatic lymph nodes in different phases

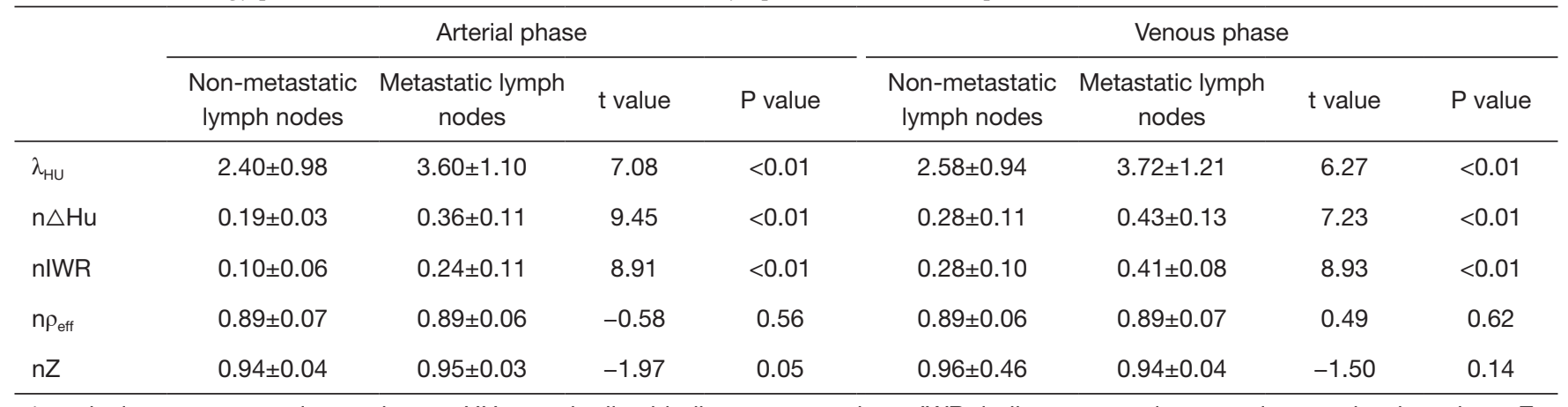

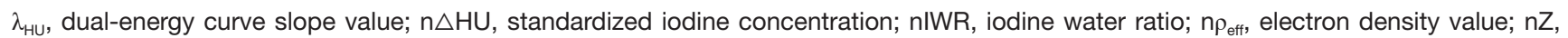
effective atom-number. 
A

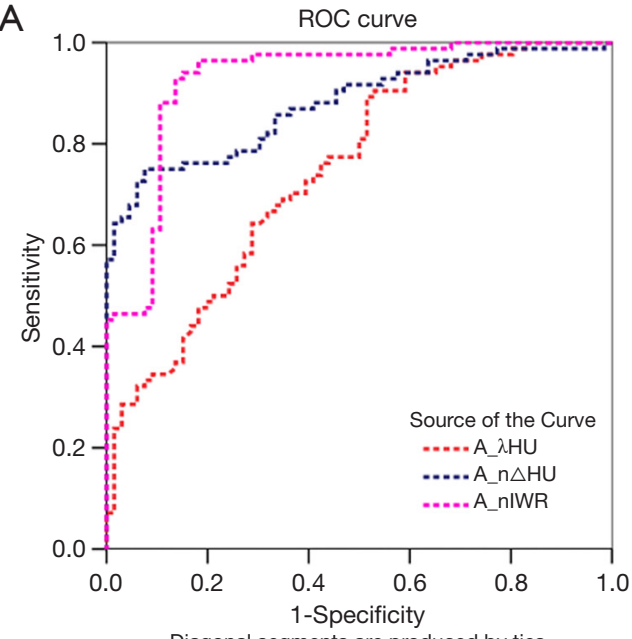

B

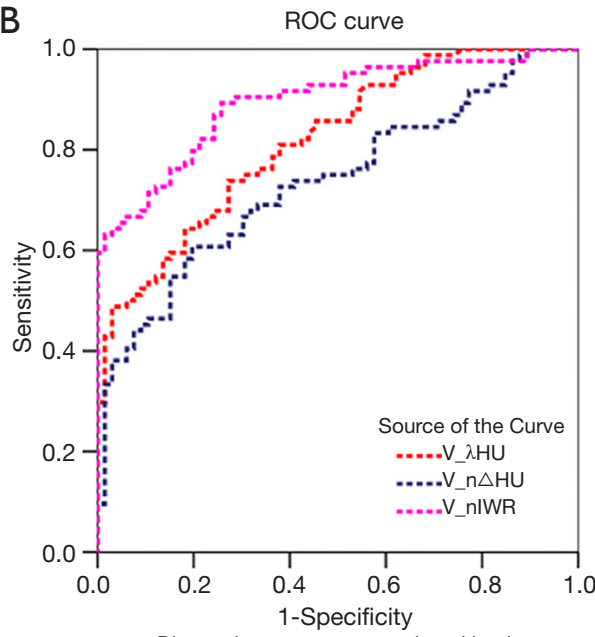

Figure 5 Diagnostic efficiency of DECT parameters.ROC curves for dual-energy parameters $\left(\lambda_{\mathrm{HU}}, \mathrm{n} \triangle \mathrm{Hu}\right.$, and $\mathrm{nIWR}$ ) in the differentiation of metastatic and non-metastatic lymph nodes. (A) ROC curves in the arterial phase: nIWR value represents the highest diagnostic efficiency, followed by $\mathrm{n} \triangle \mathrm{Hu}$ and $\lambda_{\mathrm{Hu}}$ values; (B) ROC curves in the venous phase: nIWR value displays the highest diagnostic efficiency, followed by $\lambda_{\mathrm{Hu}}$ and $\mathrm{n} \triangle \mathrm{Hu}$ values. $\mathrm{A}$, arterial phase; $\mathrm{V}$, venous phase; $\lambda \mathrm{HU}$, dual-energy curve slope value; $\mathrm{n} \Delta \mathrm{HU}$, standardized iodine concentration; nIWR, iodine water ratio.

Table 4 The value of the short axis diameter and dual-energy parameters in the arterial and venous phases $\left(\lambda_{\mathrm{HU}}\right.$, $\mathrm{n} \triangle \mathrm{HU}$, and $\left.\mathrm{nIWR}\right)$ for the differentiation of metastatic and non-metastatic lymph nodes

\begin{tabular}{|c|c|c|c|c|c|c|c|}
\hline & $\begin{array}{l}\text { Short axis } \\
\text { diameter }\end{array}$ & \multicolumn{3}{|c|}{ Arterial phase } & \multicolumn{3}{|c|}{ Venous phase } \\
\hline AUC & 0.76 & 0.80 & 0.86 & 0.88 & 0.76 & 0.79 & 0.83 \\
\hline $95 \% \mathrm{Cl}$ for $\mathrm{AUC}$ & $0.68-0.84$ & $0.74-0.85$ & $0.81-0.93$ & $0.81-0.95$ & $0.68-0.83$ & $0.72-0.85$ & $0.76-0.91$ \\
\hline Maximum Youden index & 0.46 & 0.46 & 0.65 & 0.68 & 0.38 & 0.45 & 0.52 \\
\hline Specificity (\%) & 63.64 & 65.48 & 95.24 & 95.24 & 82.14 & 91.67 & 94.05 \\
\hline Positive predictive value & 75.58 & 64.63 & 92.00 & 92.31 & 71.50 & 83.33 & 89.58 \\
\hline Negative predictive value & 70.31 & 80.88 & 80.00 & 81.63 & 70.41 & 70.30 & 77.45 \\
\hline Cut-off point & 6.81 & 3.02 & 0.19 & 0.11 & 2.56 & 2.30 & 0.28 \\
\hline
\end{tabular}

$\lambda_{\mathrm{HU}}$, dual-energy curve slope value; $\triangle \triangle \mathrm{HU}$, standardized iodine concentration; nIWR, iodine water ratio; $\mathrm{Cl}$, confidence interval; $\mathrm{AUC}$, area under the curve.

diameter of lymph nodes was similar to that of $\lambda_{\mathrm{HU}}$; however, it had the highest sensitivity and lowest specificity for differentiation. A significant difference was noted for differentiation between $\lambda_{\mathrm{HU}}$ and $\mathrm{n} \triangle \mathrm{HU}$ or between $\lambda_{\mathrm{HU}}$ and nIWR values in the arterial phase $(\mathrm{P}<0.01$, Table 5). There was also a statistical difference for differentiation between $\triangle \mathrm{HU}$ and nIWR values in the venous phase $(\mathrm{P}<0.01$, Table 5$)$.
ROC analysis was carried out to determine the threshold for each parameter to optimize both the sensitivity and specificity for differentiating normal from metastatic lymph nodes in patients with CRC. When 0.11 was used as an nIWR threshold value, the sensitivity and specificity were $71 \%$ and $95 \%$, respectively. Using an $\mathrm{n} \triangle \mathrm{HU}$ value cutoff of 0.19 , metastatic lymph nodes could be diagnosed with $70 \%$ 
Table 5 Comparison of the AUCs of the $\lambda \mathrm{HU}, \mathrm{n} \triangle \mathrm{HU}$, and $\mathrm{nIWR}$ in the arterial and venous phases

\begin{tabular}{|c|c|c|c|c|c|c|}
\hline & \multicolumn{3}{|c|}{ Arterial phase } & \multicolumn{3}{|c|}{ Venous phase } \\
\hline Difference of AUC & 0.14 & 0.18 & 0.04 & 0.08 & 0.08 & 0.16 \\
\hline Standard error & 0.05 & 0.05 & 0.04 & 0.05 & 0.05 & 0.05 \\
\hline 95\% confidence interval & $0.04-0.23$ & $0.08-0.27$ & $-0.03-0.15$ & $-0.02-0.18$ & $-0.01-0.17$ & $0.06-0.26$ \\
\hline $\mathrm{P}$ value & $<0.01$ & $<0.01$ & 0.28 & 0.1 & 0.09 & $<0.01$ \\
\hline
\end{tabular}

$\lambda_{\mathrm{HU}}$, dual-energy curve slope value; $\mathrm{n} \triangle \mathrm{HU}$, standardized iodine concentration; nIWR, iodine water ratio; AUC, area under the curve.

sensitivity and $95 \%$ specificity. When 3.02 was used as the $\lambda_{\mathrm{HU}}$ threshold value, the sensitivity and specificity were $80 \%$ and $66 \%$, respectively.

\section{Discussion}

Our study demonstrated that DECT parameters (nIWR, $\mathrm{n} \triangle \mathrm{HU}$, and $\lambda_{\mathrm{HU}}$ values in the arterial phase, especially nIWR) had a higher accuracy for differentiating nonmetastatic from metastatic nodes in CRC suggesting that DECT may be a promising technique for $\mathrm{N}$ staging in CRC.

In the present study, the nIWR, $\mathrm{n} \triangle \mathrm{HU}$, and $\lambda_{\mathrm{Hu}}$ values of metastatic lymph nodes were significantly greater than those of non-metastatic lymph nodes. Iodine concentration reflects the degree of contrast enhancement (24). An increase of iodine content in metastatic lymph nodes could be due to the increased tumor-related angiogenesis, blood volume, or vascular permeability within the metastatic lymph nodes (25). Some prior studies also found similar results in the metastatic lymph nodes from gastric (26), rectal (27), and cervical (28) cancers. In metastatic lymph nodes, the histopathological changes of metastatic foci affect blood volume and vascular osmotic pressure. There are more blood vessels in metastatic lymph nodes than non-metastatic lymph nodes (23), which results in more significant enhancement. However, a study from Liu et al. (29) reported different findings. They employed the maximal short axis and iodine content to distinguish the metastatic lymph nodes in colon cancer and found that the iodine content in metastatic lymph nodes was lower than in nonmetastatic lymph nodes. Accordingly, they believed that the number of vessels in the lymph nodes was reduced after metastasis. However, other DECT parameters, such as nIWR and $\lambda_{\mathrm{HU}}$, were not discussed in their study.

Studies on electron density and effective atom number have rarely been reported in recent years. Tatsugami et al. (30) reported that the effective atom number and electron density value might help improve accuracy in radiotherapy treatment planning. Liu et al. (23) found that the $\mathrm{nZ}$ value was significantly greater in metastatic lymph nodes than in non-metastatic lymph nodes in patients with papillary thyroid cancer. However, they did not mention electron density in their study, which might be related to the instrument and the dependent parameters. Nevertheless, in the present study, it was found that the atom number and electron density did not show significant differences in the differentiation between metastatic and non-metastatic lymph nodes, which might be associated with the difference in the primary tumor, DECT scanner, or nodal size.

Interestingly, we found that the short-axis diameter of lymph nodes showed the lowest AUC value, which was similar to $\lambda_{\mathrm{HU}}$ compared with the DECT parameters. Also, its specificity for differentiation was the lowest, whereas its sensitivity was the highest. This is in line with a previous study from Liu et al. (29), who reported the short-axis diameter of lymph nodes with the lowest specificity (46.8\%) and highest sensitivity (90.9\%) for distinguishing metastatic from non-metastatic lymph nodes. In our study, nIWR and $\mathrm{n} \triangle \mathrm{HU}$ in the arterial phase exhibited moderate sensitivity and the highest specificity compared with the short diameter of lymph nodes and other DECT parameters, reflecting their higher diagnostic accuracy for distinguishing between benign and malignant lymph nodes. Thus, DECT could be more promising for discriminating the lymph nodes status in CRC.

Moreover, Liu et al. (29) also revealed that the overall accuracy was improved from $65.7 \%$ to $82.9 \%$ with the size measurement alone when combining the normalized iodine concentration of lymph nodes with the short axis diameter. However, Fusco et al. (31) found that the linear combination 
of all morphological and/or of all functional features of dynamic enhanced MR scanning did not increase the accuracy in axillary metastatic lymph nodes discrimination in primary breast cancer. We did not evaluate the discriminative power of the combination of morphological and functional features of DECT in assessing the lymph nodes in CRC, which will be a direction for our future research.

In the present study, through ROC curve analysis, it was found that nIWR values were of significant value in differentiating metastatic from non-metastatic lymph nodes, followed by $\triangle \mathrm{Hu}$ and $\lambda_{\mathrm{HU}}$ arterial and venous phases. These values in the arterial phase showed higher diagnostic efficiency than those in the venous phase. nIWR, $\mathrm{n} \triangle \mathrm{HU}$, and $\lambda_{\mathrm{HU}}$ are related to the iodine content. After administration of contrast materials, metastatic lymph nodes demonstrated different iodine uptake from non-metastatic lymph nodes. Thus, these values in the arterial and venous phases could be useful for differentiation. This result is also supported by the findings of Tatsugami et al. (30). However, Tatsugami et al. found that the AUC of the ROC in the venous phase was greater than that in the arterial phase. They believed that the enhancement in the venous phase was caused by leakage of contrast agent into the extravascular space due to the injury of vascular endothelial cells. This meant that the venous phase was more conducive to identifying metastatic lymph nodes, which differed from our results. This discrepancy might be due to the different CT instruments used. Our study performed DECT scanning using a 320-detector-row dynamic volume CT system, which has a z-coverage width of $160 \mathrm{~mm}$ and allows for faster acquisition of the entire tumor in a single rotation without bed movement (32). IWR, $\triangle \mathrm{HU}$, and $\lambda_{\mathrm{Hu}}$ represent the change of iodine content calculated using three-material decomposition, which our CT system could more easily detect in the early stage of injection of the contrast agent.

The present study has the following limitations. Firstly, lymph nodes less than $4 \mathrm{~cm}$ in diameter were not included in this study to ensure a good one-to-one match among the in vitro and in vivo CT images. Also, measurements of smaller lymph nodes would be difficult due to the limited spatial resolution. Secondly, in the current study, the ROI was placed within each lymph node and covered the nodal parenchyma as much as possible. However, some tumor deposits are merely located in the subcapsular region of the lymph node (33), and thus, some measurements based on ROIs could have exhibited some bias. Thirdly, our study was limited to the adenocarcinoma of CRC because more than $90 \%$ of CRCs are adenocarcinomas originating from epithelial cells of the colorectal mucosa. Other rare types of CRC, such as neuroendocrine, squamous cell, adenosquamous, spindle cell, and undifferentiated carcinomas, were not included.

In summary, quantitative DECT parameters including dual-energy curve slope, iodine content, and iodine water ratio demonstrated higher accuracy than electron density value and effective atom-number to differentiate metastatic and non-metastatic lymph nodes in CRC. The iodine water ratio in the arterial phase could be more valuable for identifying metastatic nodes in CRC.

\section{Acknowledgments}

The authors thank Bing Ge, Jing Wen, Enying Zhang and Xiangxiang Chen (advanced application specialists at Canon Healthcare CT research China, Guangzhou) for their technical consultation in practicing the DECT protocol. Thanks are also due to Hansen Lin (Department of Medical Statistics, Jinan University) for providing statistical analysis. Funding: This work was supported by the Chinese Fundamental Research Funds for Central Universities (21619360) and the Guangdong University Youth Innovation Talent Program (2019KQNCX004).

\section{Footnote}

Conflicts of Interest: All authors have completed the ICMJE uniform disclosure form (available at http://dx.doi. org/10.21037/qims-20-3). The authors have no conflicts of interest to declare.

Ethical Statement: The authors are accountable for all aspects of the work in ensuring that questions related to the accuracy or integrity of any part of the work are appropriately investigated and resolved. This study was conducted in accordance with the Declaration of Helsinki (as revised in 2013), and was approved and supervised by the Medical Ethics Committee of the First Affiliated Hospital of Jinan University. All patients signed the informed consent form voluntarily before surgery.

Open Access Statement: This is an Open Access article distributed in accordance with the Creative Commons Attribution-NonCommercial-NoDerivs 4.0 International License (CC BY-NC-ND 4.0), which permits the noncommercial replication and distribution of the article with 
the strict proviso that no changes or edits are made and the original work is properly cited (including links to both the formal publication through the relevant DOI and the license). See: https://creativecommons.org/licenses/by-nc-nd/4.0/.

\section{References}

1. Cunningham D, Atkin W, Lenz HJ, Lynch HT, Minsky B, Nordlinger B, Starling N. Colorectal cancer. Lancet 2010; 375:1030-47.

2. Jemal A, Bray F, Center MM, Ferlay J, Ward E, Forman D. Global cancer statistics. CA Cancer J Clin 2011;61:69-90.

3. Torre LA, Bray F, Siegel RL, Ferlay J, Lortet-Tieulent J, Jemal A. Global cancer statistics, 2012. CA Cancer J Clin 2015;65:87-108.

4. Fan YC, Ning FL, Zhang CD, Dai DQ. Preservation versus non-preservation of left colic artery in sigmoid and rectal cancer surgery: A meta-analysis. Int J Surg 2018;52:269-77.

5. Kim J, Huynh R, Abraham I, Kim E, Kumar RR. Number of lymph nodes examined and its impact on colorectal cancer staging. Am Surg 2006;72:902-5.

6. Koca D, Binicier C, Oztop I, Yavuzsen T, Ellidokuz H, Yilmaz U. Prognostic factors affecting recurrence and survival in patients with locally advanced rectal cancer. J BUON 2012;17:291-8.

7. Zeng WG, Zhou ZX, Wang Z, Liang JW, Hou HR, Zhou HT, Zhang XM, Hu JJ. Lymph Node Ratio is an Independent Prognostic Factor in Node Positive Rectal Cancer Patients Treated with Preoperative Chemoradiotherapy Followed by Curative Resection. Asian Pac J Cancer Prev 2014;15:5365-9.

8. Miller ED, Robb BW, Cummings OW, Johnstone PA. The effects of preoperative chemoradiotherapy on lymph node sampling in rectal cancer. Dis Colon Rectum 2012;55:1002-7.

9. Backes Y, Elias SG, Bhoelan BS, Groen JN, van Bergeijk J, Seerden TCJ, Pullens HJM, Spanier BWM, Geesing JMJ, Kessels K, Kerkhof M, Siersema PD, de Vos Tot Nederveen Cappel WH, van Lelyveld N, Wolfhagen FHJ, Ter Borg F, Offerhaus GJA, Lacle MM, Moons LMG, Dutch TCRCWG. The prognostic value of lymph node yield in the earliest stage of colorectal cancer: a multicenter cohort study. BMC Med 2017;15:129.

10. Huang L, Li TJ, Zhang JW, Liu S, Fu BS, Liu W. Neoadjuvant chemotherapy followed by surgery versus surgery alone for colorectal cancer: meta-analysis of randomized controlled trials. Medicine (Baltimore) 2014;93:e231.

11. Chen Y, Yang X, Wen Z, Lu B, Xiao X, Shen B, Yu S. Fatsuppressed gadolinium-enhanced isotropic high-resolution 3D-GRE-T1WI for predicting small node metastases in patients with rectal cancer. Cancer Imaging 2018;18:21.

12. Qiu L, Liu XL, Liu SR, Weng ZP, Chen XQ, Feng YZ, Cai XR, Guo CY. Role of quantitative intravoxel incoherent motion parameters in the preoperative diagnosis of nodal metastasis in patients with rectal carcinoma. J Magn Reson Imaging 2016;44:1031-9.

13. Sun H, Xu Y, Xu Q, Shi K, Wang W. Rectal cancer: Shortterm reproducibility of intravoxel incoherent motion parameters in 3.0 T magnetic resonance imaging. Medicine (Baltimore) 2017;96:e6866.

14. Yu Z, Mao T, Xu Y, Li T, Wang Y, Gao F, Sun W. Diagnostic accuracy of dual-energy CT in gout: a systematic review and meta-analysis. Skeletal Radiol 2018;47:1587-93.

15. Zhao Y, Li X, Li L, Wang X, Lin M, Zhao X, Luo D, Li J. Preliminary study on the diagnostic value of singlesource dual-energy CT in diagnosing cervical lymph node metastasis of thyroid carcinoma. J Thorac Dis 2017;9:4758-66.

16. Jiang H, Li X. Correlation of dual-source computed tomography/dual-energy imaging with pathological grading of lung adenocarcinoma and its clinical value. Pak J Med Sci 2017;33:1429-33.

17. Li J, Fang M, Wang R, Dong D, Tian J, Liang P, Liu J, Gao J. Diagnostic accuracy of dual-energy CT-based nomograms to predict lymph node metastasis in gastric cancer. Eur Radiol 2018;28:5241-9.

18. Yang CB, Zhang S, Jia YJ, Yu Y, Duan HF, Zhang XR, Ma GM, Ren C, Yu N. Dual energy spectral CT imaging for the evaluation of small hepatocellular carcinoma microvascular invasion. Eur J Radiol 2017;95:222-7.

19. Yu Y, Lin X, Chen K, Chai W, Hu S, Tang R, Zhang J, Cao L, Yan F. Hepatocellular carcinoma and focal nodular hyperplasia of the liver: differentiation with CT spectral imaging. Eur Radiol 2013;23:1660-8.

20. Lv P, Lin XZ, Li J, Li W, Chen K. Differentiation of small hepatic hemangioma from small hepatocellular carcinoma: recently introduced spectral CT method. Radiology 2011;259:720-9.

21. George E, Wortman JR, Fulwadhva UP, Uyeda JW, Sodickson AD. Dual energy CT applications in pancreatic pathologies. Br J Radiol 2017;90:20170411.

22. Zhao Y, Li X, Li L, Wang X, Lin M, Zhao X, Luo D, Li J. Preliminary study on the diagnostic value of single- 
source dual-energy CT in diagnosing cervical lymph node metastasis of thyroid carcinoma. J Thorac Dis 2017;9:4758-66.

23. Liu X, Ouyang D, Li H, Zhang R, Lv Y, Yang A, Xie C. Papillary thyroid cancer: dual-energy spectral CT quantitative parameters for preoperative diagnosis of metastasis to the cervical lymph nodes. Radiology 2015;275:167-76.

24. Chen X, Xu Y, Duan J, Li C, Sun H, Wang W. Correlation of iodine uptake and perfusion parameters between dualenergy CT imaging and first-pass dual-input perfusion CT in lung cancer. Medicine (Baltimore) 2017;96:e7479.

25. Miles KA. Tumour angiogenesis and its relation to contrast enhancement on computed tomography: a review. Eur J Radiol 1999;30:198-205.

26. Pan Z, Pang L, Ding B, Yan C, Zhang H, Du L, Wang B, Song Q, Chen K, Yan F. Gastric Cancer Staging with Dual Energy Spectral CT Imaging. PLoS One 2013;8:e53651.

27. Al-Najami I, Lahaye MJ, Beets-Tan RGH, Baatrup G. Dual-energy CT can detect malignant lymph nodes in rectal cancer. Eur J Radiol 2017;90:81-8.

28. Naresh KN, Nerurkar AY, Borges AM. Angiogenesis is redundant for tumour growth in lymph node metastases. Histopathology 2001;38:466-70.

29. Liu H, Yan F, Pan Z, Lin X, Luo X, Shi C, Chen X, Wang B, Zhang H. Evaluation of dual energy spectral CT in

Cite this article as: Qiu L, Hu J, Weng Z, Liu S, Jiang G, Cai X. A prospective study of dual-energy computed tomography for differentiating metastatic and non-metastatic lymph nodes of colorectal cancer. Quant Imaging Med Surg 2021;11(8):3448-3459. doi: 10.21037/qims-20-3 differentiating metastatic from non-metastatic lymph nodes in rectal cancer: Initial experience. Eur J Radiol 2015;84:228-34.

30. Tatsugami F, Higaki T, Kiguchi M, Tsushima S, Taniguchi A, Kaichi Y, Yamagami T, Awai K. Measurement of electron density and effective atomic number by dualenergy scan using a 320-detector computed tomography scanner with raw data-based analysis: a phantom study. J Comput Assist Tomogr 2014;38:824-7.

31. Fusco R, Sansone M, Granata V, Di Bonito M, Avino F, Catalano O, Botti G, Petrillo A. Use of Quantitative Morphological and Functional Features for Assessment of Axillary Lymph Node in Breast Dynamic ContrastEnhanced Magnetic Resonance Imaging. Biomed Res Int 2018;2018:2610801.

32. Imae T, Haga A, Nakagawa K, Ino K, Tanaka K, Okano Y, Sasaki K, Saegusa S, Shiraki T, Oritate T, Yano K, Shinohara H. Motion Analysis of Target in Stereotactic Radiotherapy of Lung Tumors Using 320-row Multidetector CT. Nihon Hoshasen Gijutsu Gakkai Zasshi 2011;67:202-11.

33. Rizzo S, Radice D, Femia M, De Marco P, Origgi D, Preda L, Barberis M, Vigorito R, Mauri G, Mauro A, Bellomi M. Metastatic and non-metastatic lymph nodes: quantification and different distribution of iodine uptake assessed by dual-energy CT. Eur Radiol 2018;28:760-9. 